This is the final peer-reviewed accepted manuscript of:

2015. Structure-morphology correlation in electrospun fibers of semicrystalline polymers by simultaneous synchrotron SAXS-WAXD. pp.154-163. In POLYMER - ISSN:0032-3861 vol. 63 Gazzano, M; Gualandi, C.; Zucchelli, A.; Sui, T.; Korsunsky, A.M.; Reinhard, C.; Focarete, M.L.

The final published version is available online at: $\mathrm{http}: / / \mathrm{dx} . \mathrm{doi} . \mathrm{org} / 10.1016 / \mathrm{j}$. polymer.2015.03.002

Rights / License:

The terms and conditions for the reuse of this version of the manuscript are specified in the publishing policy. For all terms of use and more information see the publisher's website.

This item was downloaded from IRIS Università di Bologna (https://cris.unibo.it/)

When citing, please refer to the published version. 


\title{
Structure-morphology correlation in electrospun fibers of semicrystalline polymers by simultaneous synchrotron SAXS-WAXD
}

\author{
M. Gazzano ${ }^{\text {a, }{ }^{*}, \text { C. Gualandi }}{ }^{\text {b }}$, A. Zucchelli ${ }^{\text {c }}$, T. Sui ${ }^{\text {d }}$, A.M. Korsunsky ${ }^{\text {d, e }}$, C. Reinhard ${ }^{\text {e }}$, \\ M.L. Focarete ${ }^{b, * *}$ \\ a ISOF - CNR, via Gobetti 101, 40129 Bologna, Italy \\ b Department of Chemistry “G. Ciamician”, University of Bologna, via Selmi 2, 40126 Bologna, Italy \\ ${ }^{c}$ Department of Industrial Engineering, University of Bologna, Viale Risorgimento 2, 40136 Bologna, Italy \\ ${ }^{\mathrm{d}}$ Multi-Beam Laboratory for Engineering Microscopy (MBLEM), Department of Engineering Science, University of Oxford, Parks Road, Oxford OX1 3PJ, UK \\ e Diamond Light Source, Harwell Science and Innovation Campus, Didcot, OX11 ODE, UK
}

\begin{abstract}
A B S T R A C T
Simultaneous SAXS-WAXD measurements are carried out to gain information about the microstructure and the molecular orientation developed by polymeric chains during the electrospinning process. Three semicrystalline polymers were studied, namely polyacrylonitrile, Nylon 6,6, and poly(ethylene oxide), as non-woven mats with either randomly arranged or aligned electrospun fibers. Mat thermal and morphological properties are investigated, together with their structural details in order to derive their hierarchical structure from the macro to the nano-scale. SAXS patterns have an elliptical shape with the main axis along the equator direction. No reflections are noticeable along the meridional direction, suggesting that the investigated electrospun fibers have a fibrillar structure with no trace of lamellar morphology. Combining the values of the unit cell and of the crystal size it can be concluded that in the fibers the ordered domains are organized into a bundle of fibrils due to the lateral aggregation of roughly 10 unit cells and the regular ordering of about either 50 (Nylon 6,6 and polyacrylonitrile) or 25 (poly(ethylene oxide)) cells in the chain direction.
\end{abstract}

\section{Introduction}

Electrostatic spinning has been recognized as a powerful technique for the production of ultrafine fibers (with diameters ranging from a few hundreds of nanometers to tenths of micrometers) with unique properties and many potential applications in diverse fields $[1,2]$. Structural orientation and crystallinity in electrospun nanofibers are determined not only by the chemistry of the polymer and the nature of the polymeric solution, but also by the processing parameters governing the process. Indeed, it was demonstrated that the particular feature of the electrospinning process, namely the presence of strong elongation force, is responsible for the strong diameter reduction within the jet that results in the formation of an ultrafine fiber. This process has a great effect on the

\footnotetext{
* Corresponding author. Tel.: +39 051 2099552; fax: +39 0512099456.

** Corresponding author. Tel.: +39 051 2099572; fax: +39 0512099456.

E-mail addresses: gazzano@isof.cnr.it (M. Gazzano), c.gualandi@unibo.it (C. Gualandi), a.zucchelli@unibo.it (A. Zucchelli), tan.sui@eng.ox.ac.uk (T. Sui), alexander.korsunsky@eng.ox.ac.uk (A.M. Korsunsky), christina.reinhard@diamond. ac.uk (C. Reinhard), marialetizia.focarete@unibo.it (M.L. Focarete).
}

structural elements constituting the fibers, i.e. on the macromolecular assemblies present inside the fibers, in particular on their macromolecular orientation, chain conformation and crystal structure $[3,4]$. In fact, it is well known that during the electrospinning process, characterized by high strain rates of the order of $10^{4} \mathrm{~s}^{-1}$ [5], segments of the macromolecules in the liquid phase show different degree of alignment of their chains in the flow direction, i.e. along the fiber axis, whilst rapid solvent evaporation occurs. However, the effective persistence of chain orientation within the final solid fibers depends on the balance between chain relaxation time and the rate of solvent evaporation. Indeed, the oriented chains can relax at the end of the stretching phase, especially when the electrospun nanofibers, collected as a mat, still contain a small amount of solvent. Polymer parameters such as the molecular weight, architecture (linear or branched macromolecules) and chain flexibility strongly affect the chain relaxation time.

The general topic of molecular orientation in polymers induced by mechanical drawing and other processing technologies has been intensively studied by means of various techniques such as birefringence, fluorescence polarization, diffraction, infrared dichroism spectroscopy, NMR spectroscopy [6-8]. Referring in particular to 
electrospun fibers, commonly employed methods to investigate their internal structural organization are polarized infrared spectroscopy (IR) [9-11] and diffraction techniques such as Wide Angle X-ray diffraction (WAXD) [10,12-14], Small Angle X-ray scattering (SAXS) [12-14] as well as simultaneous SAXS-WAXD techniques [15]. Diffraction techniques, however, are usually applied to the analysis of the crystalline phase of polymeric materials, whereas they are of limited interest for fibers of amorphous or low crystalline polymers. All these techniques require a relatively high amount of fibrous material in order to obtain acceptable signal-to-noise ratios. Moreover, in order to study the molecular orientation within the fiber, the electrospun mat should display highly aligned fibers. Other techniques, such as Atomic Force Microscopy (AFM) [16,17], and Selected Area Electron Diffraction (SAED) [18-20] were also applied to characterize molecular orientation and structural characteristics of individual fibers. In particular, SAED was recently employed to determine the molecular orientation and crystal morphology in polyethylene fibers with different diameters and it was found that thinner fibers possessed higher degree of orientation [19]. However, the information provided by this technique remains restricted to the crystal phase. Recently, confocal Raman spectroscopy applied to single nanofibers was shown to offer many advantages since, beside providing information about conformation and crystallinity at the molecular level, it allows to distinguish between the molecular chain orientation of the crystalline and amorphous phases [21,22].

Despite the great research effort invested up to date, the investigation of the influence of the electrospinning processing conditions on macromolecular orientation, chain conformation and crystal structure still remains a research theme of great interest within the scientific community, since better understanding of the correlation between the structural characteristics and the electrospinning processing conditions will help to control the physical and mechanical properties of the final products.

In this work we report the use of simultaneous SAXS/WAXD measurements using synchrotron radiation to gain further knowledge of the microstructure development occurring during the electrospinning process. Three important examples of semicrystalline engineering thermoplastic polymers with different chemical structure were chosen as model systems, namely, polyacrylonitrile (PAN), Nylon 6,6 (NYL) and poly(ethylene oxide) (PEO). These polymers possess linear chains with different chain flexibility: PEO is a highly crystalline polymer and it displays the highest chain flexibility with a glass transition temperature, $T_{g}$, around $-50{ }^{\circ} \mathrm{C}$, followed by NYL $\left(\mathrm{T}_{\mathrm{g}}=50{ }^{\circ} \mathrm{C}\right)$, that crystallizes thanks to its regular chain structure with amide groups forming intermolecular hydrogen bonds. PAN chains are the less flexible, with a $T_{g}$ around $100{ }^{\circ} \mathrm{C}$, and they are characterized by the presence of nitrile groups that are able to generate polar interactions. Despite being atactic, PAN displays a significant amount of crystallinity due to the formation of crystals from mixed tacticity chains, in which the isotactic sequences cocrystallize with syndiotactic units [23]. Non-woven mats with both randomly arranged and aligned electrospun fibers were fabricated, and mat thermal and morphological properties were investigated, together with their structural details that could be evaluated by taking advantage of the high intensity of the synchrotron radiation X-ray source (Diamond Light Source, UK).

\section{Experimental part}

\subsection{Materials}

Poly(ethylene oxide) (PEO, $\mathrm{M}_{\mathrm{V}} \sim 1 \times 10^{6} \mathrm{~g} / \mathrm{mol}$ ) and Polyacrylonitrile (PAN, $\mathrm{M}_{\mathrm{w}}=1.5 \times 10^{5} \mathrm{~g} / \mathrm{mol}$ ) were purchased from Sigma-Aldrich. Nylon 6,6 (NYL, Zytel ${ }^{\circledR}$ E53 NC010) was kindly provided by DuPont. Dimethylformamide (DMF), Formic Acid (FA) and Chloroform (CLF) were purchased from Sigma Aldrich and were used without further purification.

\subsection{Sample preparation}

Electrospun meshes were produced by using an in-house electrospinning apparatus composed of a high voltage power supply (Spellman, SL 50 P 10/CE/230), a syringe pump (KD Scientific 200 series), a glass syringe, a stainless-steel blunt-ended needle (inner diameter $=0.84 \mathrm{~mm}$ ) connected with the power supply electrode, and a grounded high-speed rotating collector (length $=12 \mathrm{~cm}$, diameter $=5 \mathrm{~cm}$ ). The polymer solution was dispensed, through a Teflon tube, to the needle that was vertically placed over the collecting mandrel. Fibers were collected with a random arrangement on the drum rotating at a speed of $5.2 \mathrm{~m} / \mathrm{s}$, whereas aligned fibers were obtained by increasing the rotational speed to $16.2 \mathrm{~m} / \mathrm{s}$. Electrospun meshes of PEO were obtained by dissolving the polymer in MilliQ water at a concentration of $4 \% \mathrm{w} / \mathrm{v}$ and by using the following electrospinning conditions: needle-to-collector distance $=20 \mathrm{~cm}$, applied voltage $=13 \mathrm{kV}$ and flow rate $=0.3 \mathrm{ml} /$ h. PAN was dissolved in DMF at a concentration of $7.5 \% \mathrm{w} / \mathrm{v}$ and it was electrospun by using needle-to-collector distance $=20 \mathrm{~cm}$, applied voltage $=19 \mathrm{kV}$ and flow rate $=0.9 \mathrm{ml} / \mathrm{h}$. NYL was dissolved in CLF:FA $=50: 50(\mathrm{v} / \mathrm{v})$ at a concentration of $14 \% \mathrm{w} / \mathrm{v}$ and it was electrospun by using needle-to-collector distance $=10 \mathrm{~cm}$, applied voltage $=22 \mathrm{kV}$ and flow rate $=0.3 \mathrm{ml} / \mathrm{h}$. All electrospun meshes were produced at room temperature (RT) and relative humidity in the range $45-55 \%$. Electrospun membranes were kept under vacuum at RT overnight to remove residual solvents.

\subsection{Characterization methods}

Fiber morphology was observed with a Philips 515 scanning electron microscope (SEM) at an accelerating voltage of $15 \mathrm{kV}$. Prior to SEM analysis, samples were sputter-coated with gold. Fiber diameter distribution was evaluated by measuring 200 fiber diameters with an image acquisition software (EDAX Genesis), and results were reported as the mean \pm standard deviation (SD). The relative degree of fiber alignment was measured by processing the SEM images with Image $2 D$ fast Fourier transform (2D FFT) function and by applying an oval profile plug-in (designed by Bill O'Connell), as previously described in detail by Ayres et al. [24]. In brief, grayscale 8 -bit images were cropped to $764 \times 764$ pixels and processed using 2D FFT. The resulting FFT output image was rotated $90^{\circ}$ to correct for the inherent rotation of the data induced by $2 \mathrm{D}$ FFT analysis. The application of the oval profile plug-in enabled the summing of pixel intensities along the radius for each angle of a circular projection, which was previously placed on the FFT output image by using the ImageJ circular marquee tool. The summed pixel intensities were normalized to a baseline value of 0 and plotted as the function of the corresponding angle in arbitrary units from $0^{\circ}$ to $180^{\circ}$. The height of the resulting peak corresponds to the degree of fiber alignment. Differential Scanning Calorimetry (DSC) measurements were carried out using a TA Instruments Q100 DSC equipped with the Liquid Nitrogen Cooling System accessory. DSC scans of electrospun membranes were performed in helium atmosphere from $-80^{\circ} \mathrm{C}$ to $120^{\circ} \mathrm{C}$ for PEO membranes, from $-50{ }^{\circ} \mathrm{C}$ to $300{ }^{\circ} \mathrm{C}$ for NYL membranes and from $-30{ }^{\circ} \mathrm{C}$ to $410{ }^{\circ} \mathrm{C}$ for PAN membranes at a heating scan rate of $20{ }^{\circ} \mathrm{C} / \mathrm{min}$. The crystallinity degree, $\chi_{c}$, was calculated by applying Equation (1):

$\chi_{\mathrm{c}}=\frac{\Delta \mathrm{H}_{\mathrm{m}}}{\Delta \mathrm{H}_{\mathrm{m}}^{0}} \cdot 100$ 
where $\Delta \mathrm{H}_{\mathrm{m}}$ is the experimental endothermic heat of fusion of the investigated sample and $\Delta \mathrm{H}^{0}{ }_{\mathrm{m}}$ is the endothermic heat of fusion of the $100 \%$ crystalline polymer (PEO $\Delta \mathrm{H}^{0}{ }_{\mathrm{m}}=196.6 \mathrm{~J} / \mathrm{g}$; NYL $\left.\Delta \mathrm{H}_{\mathrm{m}}^{0}=255.4 \mathrm{~J} / \mathrm{g}[25]\right)$.

\subsection{X-ray data collection}

X-ray diffraction data were collected at the I12-JEEP beam line of the Diamond Light Source (Didcot, Oxfordshire, UK) by using a monochromatic beam of $53 \mathrm{KeV}(\lambda=0.2330 \AA)$ with a spot size of $500 \mu \mathrm{m} \times 700 \mu \mathrm{m}$ at the sample position. The sample was scanned through the beam by a computer-controlled $x-y$ translation table. In order to avoid beam damage of the sample, new material was presented to the beam at each exposure: typically 100 exposures were collected for $2 \mathrm{~s}$ each in a linear scan with a $500 \mu \mathrm{m}$ step. The diffraction patterns were collected using Pixium FE4343F detector positioned $2030 \mathrm{~mm}$ downstream from the sample. A matrix of $2880 \times 2881$ pixels $(149 \mu \mathrm{m} \times 149 \mu \mathrm{m} /$ each $)$ was recorded at each exposure. Mesh samples were carefully folded 10 times to preserve orientation and compacted by compressing in the direction perpendicular to the sheet extension to increase the sample volume illuminated by the radiation and to reduce the presence of microvoids between the fibers. Extreme care was used during folding of aligned samples in order to prevent any increase in fiber misalignment due to imperfect mesh layers overlapping.

\subsection{X-ray data analysis}

Data display, background subtraction, data summation and data merging were carried out by the Fit2d routine [26]. The 100 patterns from each sample were merged before analysis. Radial and azimuthal scans were plotted by means of Fit2d. The crystallinity degree $\left(\chi_{c}\right)$ was calculated from the diffraction profiles by the ratio between the crystalline diffraction area $\left(A_{c}\right)$ and the total area of the diffraction profile $\left(A_{t}\right), \chi_{c}=A_{c} / A_{t}$. The crystalline diffraction area was obtained from the total area of the diffraction profile by subtracting the amorphous halo. The non-coherent scattering was taken into consideration. The program Scatter [27] was employed in the analysis of non-aligned samples data.

\section{Results and discussion}

\subsection{Morphological properties}

The three semicrystalline polymers investigated in this work, i.e. PEO, PAN and NYL, were processed through electrospinning in order to fabricate nonwoven fibrous mats with either randomly arranged or highly aligned fibers. Optimized processing conditions for each polymer (reported in the experimental section) allowed to obtain regular defect-free fibers with uniform diameters, as shown by the SEM micrographs reported in Fig. $1 \mathrm{~A}$ and B, Fig. 2A and B and Fig. $3 A$ and $B$ for PEO, NYL and PAN, respectively. All electrospun samples display fiber diameters in the sub-micrometric range, the NYL fibers being the smallest ones whereas PAN fibers show the biggest cross section (Table 1 ). The FFT frequency distributions reported in the insets of Figs. 1B, 2B and 3B provide a measure of the degree of fiber alignment. The height and overall shape of the peaks centered at $90^{\circ}$ demonstrate that fibers in the aligned mats display a comparable degree of alignment. It is worth nothing that, for each polymer, aligned fibers display lower diameters than the corresponding random ones. The decrease of fiber diameter is probably due to further stretching experienced by the fibers when drawn onto the high velocity rotating drum, as previously found by other authors [11].
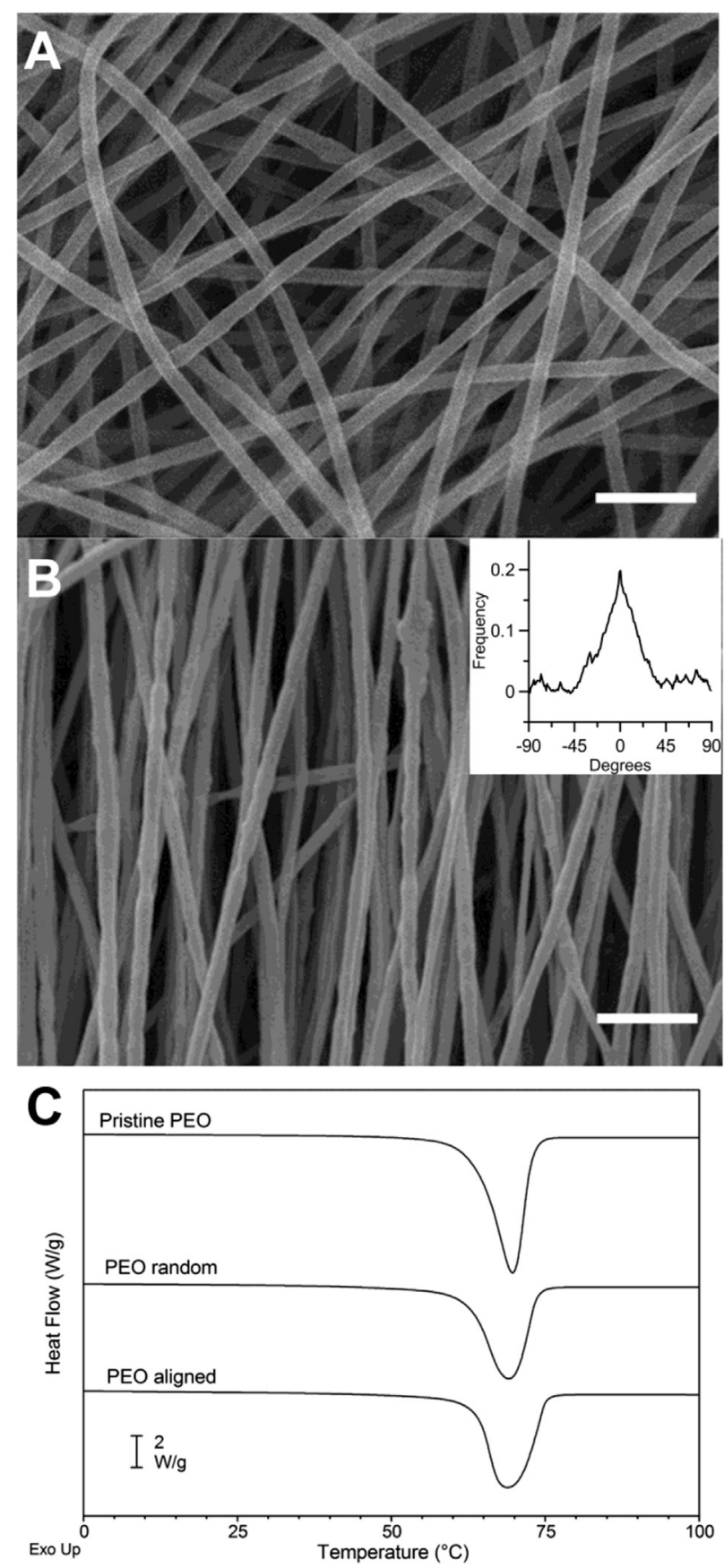

Fig. 1. SEM micrographs of PEO fibers, random (A) and aligned (B), bar $=2 \mu \mathrm{m}$; inset: 2D FFT frequency distribution of the corresponding SEM image. In (C) DSC scans of PEO powder (top), PEO random (middle) and PEO aligned (bottom) fibers.

\section{Thermal behavior}

Fig. 1C displays the DSC curves of PEO samples in the form of powder and electrospun mats. PEO samples are semicrystalline materials with the melting temperature $\left(\mathrm{T}_{\mathrm{m}}\right)$ around $70^{\circ} \mathrm{C}$. Among PEO samples, the pristine one shows the highest value of the enthalpy of fusion, followed by the fibers of the aligned PEO mats, and PEO fibers of the random mats (see Table 2). These data demonstrate that electrospun samples possess a lower amount of crystal phase with respect to the pristine material. Indeed, the crystallinity degree $\left(\chi_{c}\right)$ calculated by DSC data is $90 \%$ for the pristine polymer and $78 \%$ and $74 \%$ for the aligned and random 

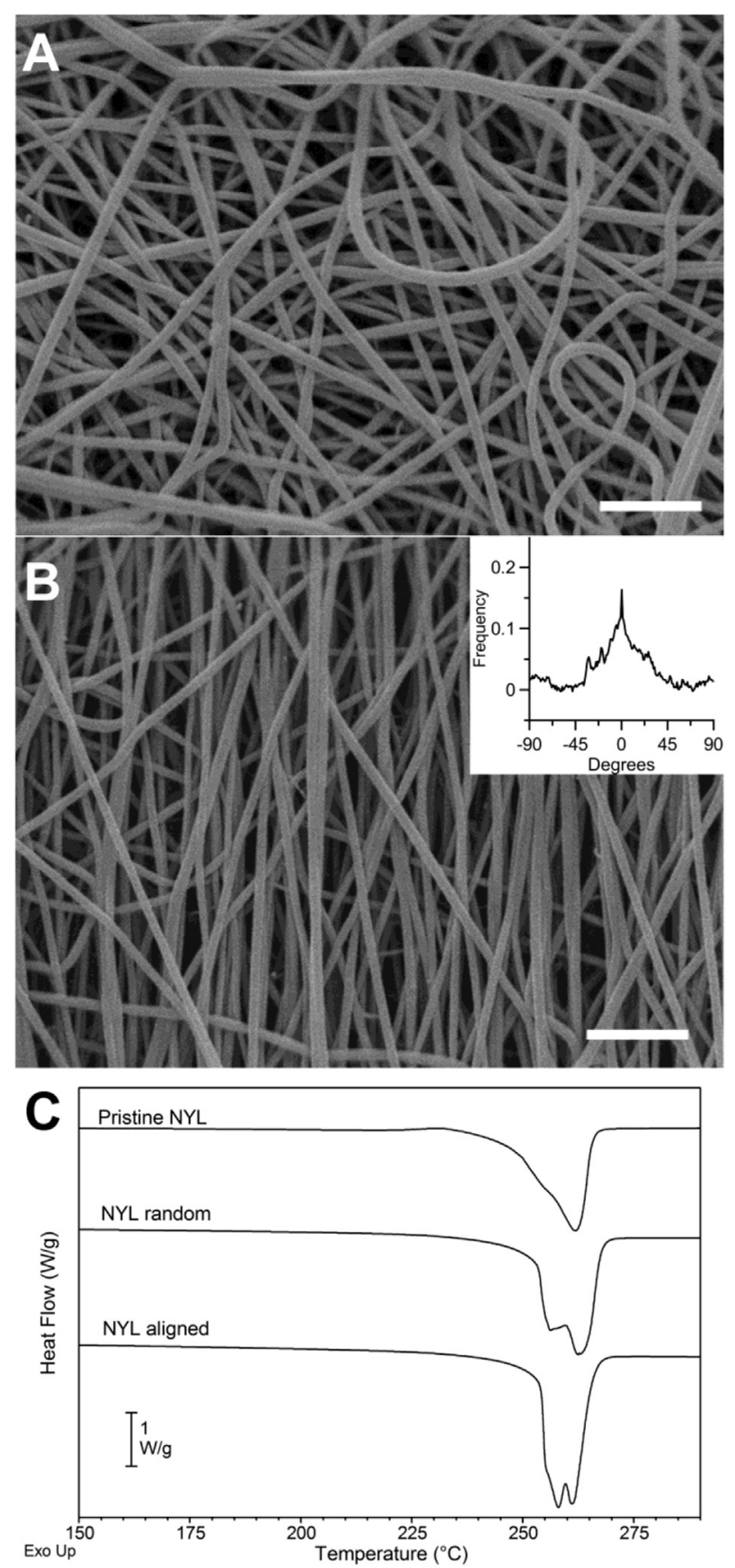

Fig. 2. SEM micrographs of NYL fibers, random (A) and aligned (B), bar $=2 \mu \mathrm{m}$; inset: 2D FFT frequency distribution of the corresponding SEM image. In (C) DSC scans of NYL grains (top), NYL random (middle) and NYL aligned (bottom) fibers.

fibers, respectively. A significant decrease in the crystal fraction or even total inhibition of crystallization when electrospinning crystallizable polymers has been previously reported [28-30], and it was attributed to the fast solvent evaporation, occurring concurrently with crystallization, that limits the formation of crystal phase and is expected to have a strong influence over the crystallization kinetics [31-33]. Indeed, whereas the strong extensional forces experienced by the macromolecular chains during the electrospinning process promote the formation of an oriented phase, the fast solvent evaporation restricts chain mobility and, depending on the crystallization kinetics of the polymer, may prevent the formation of the regularly structured crystalline phase. For aligned
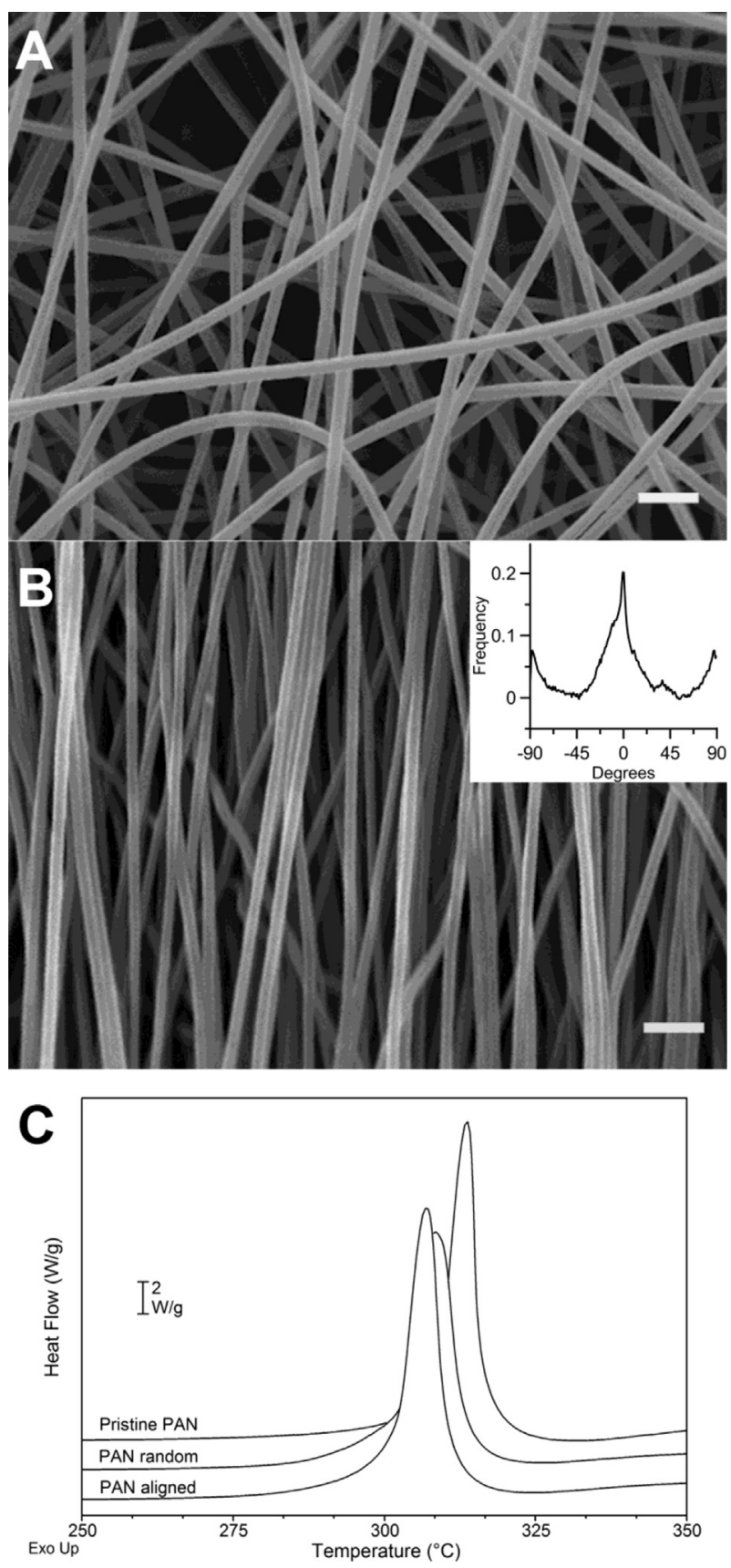

Fig. 3. SEM micrographs of PAN fibers, random (A) and aligned (B), bar $=2 \mu \mathrm{m}$; inset: 2D FFT frequency distribution of the corresponding SEM image. In (C) DSC scans of PAN powder (top), PAN random (middle) and PAN aligned (bottom) fibers.

samples, however, it might be expected that the additional stretching of the macromolecules being collected onto the high velocity rotating drum, in parallel with the reduction of the fiber diameter, facilitate polymer crystallization and the orientation of the polymer chains in the direction of the fiber axis [10]. Our results confirm this point, since the crystallinity degree of aligned PEO fibers is higher compared to that of random fibers $\left(\chi_{c}=78 \%\right.$ vs $\chi_{\mathrm{c}}=74 \%$ ).

Fig. 2C displays DSC curves of NYL samples. NYL is a semicrystalline polymer with a high $\mathrm{T}_{\mathrm{m}}$ (around $260{ }^{\circ} \mathrm{C}$ ). The doublepeak melting endotherm can be associated with the melting and recrystallization of crystals with different morphology and perfection. The pristine sample shows a lower enthalpy of fusion 
Table 1

Morphological and microstructural parameters.

\begin{tabular}{|c|c|c|c|c|c|c|c|c|}
\hline Sample & $\begin{array}{l}\text { Fiber diam. } \\
(\mathrm{nm})\end{array}$ & $\begin{array}{l}\text { FWHM } \\
\left({ }^{\circ}\right)^{\mathrm{b}}\end{array}$ & $h k l^{c}$ & $\begin{array}{l}\text { C.S. } \\
(\mathrm{nm})\end{array}$ & $\begin{array}{l}\mathrm{H}^{\mathrm{e}} \\
\left({ }^{\circ}\right)\end{array}$ & $\begin{array}{l}\Pi^{\mathrm{f}} \\
(\%)\end{array}$ & $\begin{array}{l}B_{\phi}{ }^{g} \\
\left({ }^{\circ}\right)\end{array}$ & $\begin{array}{l}\mathrm{L}_{\mathrm{f}}^{\mathrm{h}} \\
(\mathrm{nm})\end{array}$ \\
\hline \multirow[t]{2}{*}{ PEO random } & $290 \pm 30$ & 0.10 & 120 & 13.4 & & & & \\
\hline & & $0.22^{\mathrm{i}}$ & 032 & - & & & & \\
\hline \multirow[t]{2}{*}{ PEO aligned } & $240 \pm 50$ & 0.10 & 120 & 13.4 & 60 & 67 & 0.6 & 54 \\
\hline & & $0.21^{\mathrm{i}}$ & 032 & - & 82 & 55 & & \\
\hline \multirow[t]{2}{*}{ NYL random } & $170 \pm 30$ & 0.28 & 100 & 4.8 & & & & \\
\hline & & 0.32 & 010 & 4.2 & & & & \\
\hline \multirow[t]{2}{*}{ NYL aligned } & $160 \pm 30$ & 0.27 & 100 & 5.0 & 48 & 73 & 0.7 & 93 \\
\hline & & 0.31 & 010 & 4.3 & 50 & 72 & & \\
\hline PAN random & $420 \pm 50$ & 0.28 & 010 & 4.8 & & & & \\
\hline PAN aligned & $370 \pm 60$ & 0.30 & 010 & 4.5 & 75 & 58 & 0.5 & 78 \\
\hline
\end{tabular}

a Fiber diameter distribution from SEM observations ( \pm standard deviation).

b Full Width at Half Maximum, from equatorial scan.

c Miller indexes of crystallographic planes.

d Crystal Size estimated by Scherrer equation.

e Full Width at Half Maximum, from azimuthal scan taken as mean value of the two equivalent equatorial reflections.

${ }^{f}$ Parallelism degree, $\Pi=100 \times(180-\mathrm{H}) / 180$.

g $B_{\Phi}=$ misorientation width, from SAXS data.

${ }^{\mathrm{h}} \mathrm{L}_{\mathrm{f}}=$ fibril length, from SAXS data.

i Due to several overlapped reflections $(032,-132,-212)$, not suitable for C.S. measurement.

compared to the random electrospun mats, while aligned fibers display the highest value of $\Delta \mathrm{H}_{\mathrm{m}}$, and thus the highest degree of crystallinity (see Table 2). Contrary to the relationship that was observed in the case of PEO samples, electrospinning of NYL induces the formation of a higher amount of crystal phase compared to the pristine material, as previously found by other authors [3]. The crystallinity degree calculated by DSC data is $26 \%$ for the pristine polymer and $34 \%$ and $31 \%$ for the aligned and random fibers, respectively. This behavior can be related to the chemical structure of the NYL macromolecule, with amide groups forming intermolecular hydrogen bonds that are a further driving force for the orientation and crystallization during fiber processing. NYL macromolecules experiencing strong elongational forces readily adopt the conformation they possess within a crystal: the fully extended planar conformation. As a consequence, the formation of crystals is an 'assisted process' during the electrospinning process. Again, the aligned fibers contain a higher amount of crystal phase compared to the random ones $\left(\chi_{c}=34 \%\right.$ vs $\left.\chi_{c}=31 \%\right)$.

The DSC heating scans of PAN samples are reported in Fig. 3C. A strong exothermic peak in the temperature range $275-325^{\circ} \mathrm{C}$ was detected for all PAN samples. This peak has been associated to the cyclization reaction undergone by PAN molecules to give conjugated carbon-nitrogen sequences [34]. The exothermal heat $\left(\Delta \mathrm{H}_{\text {exo }}\right)$ associated with this reaction increases in electrospun fibers with respect to pristine PAN, and is higher in the case of aligned fibers (pristine PAN: $\Delta \mathrm{H}_{\mathrm{exo}}=385 \mathrm{~J} / \mathrm{g}$; PAN random mat: $\Delta \mathrm{H}_{\mathrm{exo}}=392 \mathrm{~J} / \mathrm{g}$; PAN aligned mat: $\Delta \mathrm{H}_{\mathrm{exo}}=401 \mathrm{~J} / \mathrm{g}$ ). Concomitantly, the peak temperature decreases in electrospun samples, with the lower value found in the case of PAN aligned fibers (pristine PAN: $\mathrm{T}_{\text {peak }}=314^{\circ} \mathrm{C}$; PAN random: $\mathrm{T}_{\text {peak }}=309{ }^{\circ} \mathrm{C}$; PAN aligned: $\mathrm{T}_{\text {peak }}=307{ }^{\circ} \mathrm{C}$ ). The increase of exothermal heat and the concomitant decrease of the temperature associated to the cyclization reaction from pristine PAN to PAN random and PAN aligned can be related to the increase of macromolecular orientation in the different samples that leads to a more efficient cyclization reaction. Similar results were found after hot-stretching PAN electrospun fibers to increase polymer crystallinity [35,36] and by Gu et al. [37] when comparing DSC curves of PAN in form of film and electrospun fibers.

Altogether, DSC results of the investigated samples demonstrate that polymer chemistry has a great effect on the crystallization behavior of the electrospun fibers. Both in the case of NYL and PAN the electrospun samples show higher crystallinity than the corresponding pristine material, while the opposite result was found for PEO. These findings suggest that fiber drawing favors the increase of the crystal phase thanks to the presence of intra- and intermolecular interactions in the case of NYL and PAN, that in turns contribute to locking the molecules in a packed conformation. In the case of PEO the decrease of crystallinity from the pristine sample to the electrospun fibers can be explained considering the high chain flexibility $\left(T_{g}=-50^{\circ} \mathrm{C}\right)$ that determines the fast relaxation of the stretched chains concomitant with the fast solvent evaporation. Moreover, for the same polymeric material, it was found that the collection mode of the fibers has an influence on the fiber diameter and on the amount of the obtained crystal phase. The collection on the high speed rotating drum determines an increase in the amount of crystal phase in the aligned fibers with respect to the random ones, due to the additional stretching undergone by the macromolecules collected on the drum that concomitantly induces an increased molecular orientation and the reduction of the fiber diameter.

\subsection{Structural characterization}

The combined WAXD and SAXS diffraction patterns of the electrospun PEO, NYL and PAN samples are shown in Figs. 4-6. All samples show several wide angle reflections above a background hump that is usual for semicrystalline materials. A diffusion halo is observed in the small angle region. The patterns of the aligned samples display the same reflections as the corresponding random ones, suggesting that the crystal phase formed is identical, irrespective of fiber alignment degree. The crystallinity degree of the different samples were calculated from WAXD data and are reported in Table 2, together with those calculated from DSC analysis. WAXD enables to confirm that: (i) $\chi_{c}$ is higher when the polymer is in form of electrospun fibers with respect to the pristine sample for NYL and PAN and (ii) the aligned fibers display higher crystallinity than the random ones. In the case of PAN, the latter result is in line with the change of the exothermal peak associated to the cyclization reaction occurring during DSC that was associated to the increase of macromolecular orientation.

By considering the WAXD patterns of the aligned and random fibers differences emerge when comparing the intensities of diffraction peaks. In aligned samples the intensity of the two most

Table 2

Melting enthalpy and crystallinity degree $\left(\chi_{\mathrm{c}}\right)$, calculated by DSC and WAXD, of the investigated samples.

\begin{tabular}{|c|c|c|c|c|c|c|c|c|c|}
\hline \multirow[t]{2}{*}{ Sample } & \multicolumn{3}{|c|}{ PEO } & \multicolumn{3}{|c|}{ NYL } & \multicolumn{3}{|c|}{ PAN } \\
\hline & $\Delta \mathrm{H}_{\mathrm{m}}(\mathrm{J} / \mathrm{g})$ & $\chi_{\mathrm{c}}(\%) \mathrm{DSC}^{\mathrm{a}}$ & $\chi_{\mathrm{c}}(\%) \mathrm{WAXD}^{\mathrm{b}}$ & $\Delta \mathrm{H}_{\mathrm{m}}(\mathrm{J} / \mathrm{g})$ & $\chi_{c}(\%) D C^{a}$ & $\chi_{\mathrm{c}}(\%) \mathrm{WAXD}^{\mathrm{b}}$ & $\Delta \mathrm{H}_{\mathrm{m}}(\mathrm{J} / \mathrm{g})$ & $\chi_{\mathrm{c}}(\%) \mathrm{DSC}^{\mathrm{a}}$ & $\chi_{c}(\%)$ WAXD $^{\mathrm{b}}$ \\
\hline Pristine & 176 & 90 & 81 & 67 & 26 & 36 & n.a. ${ }^{\mathrm{c}}$ & n.a. ${ }^{c}$ & 39 \\
\hline Aligned & 153 & 78 & 79 & 87 & 34 & 44 & n.a. ${ }^{c}$ & n.a. ${ }^{c}$ & 49 \\
\hline Random & 145 & 74 & 77 & 80 & 31 & 42 & n.a. ${ }^{c}$ & n.a. ${ }^{c}$ & 48 \\
\hline
\end{tabular}

a $\pm 2 \%$.

b $\pm 5 \%$.

c n.a. = not available. 

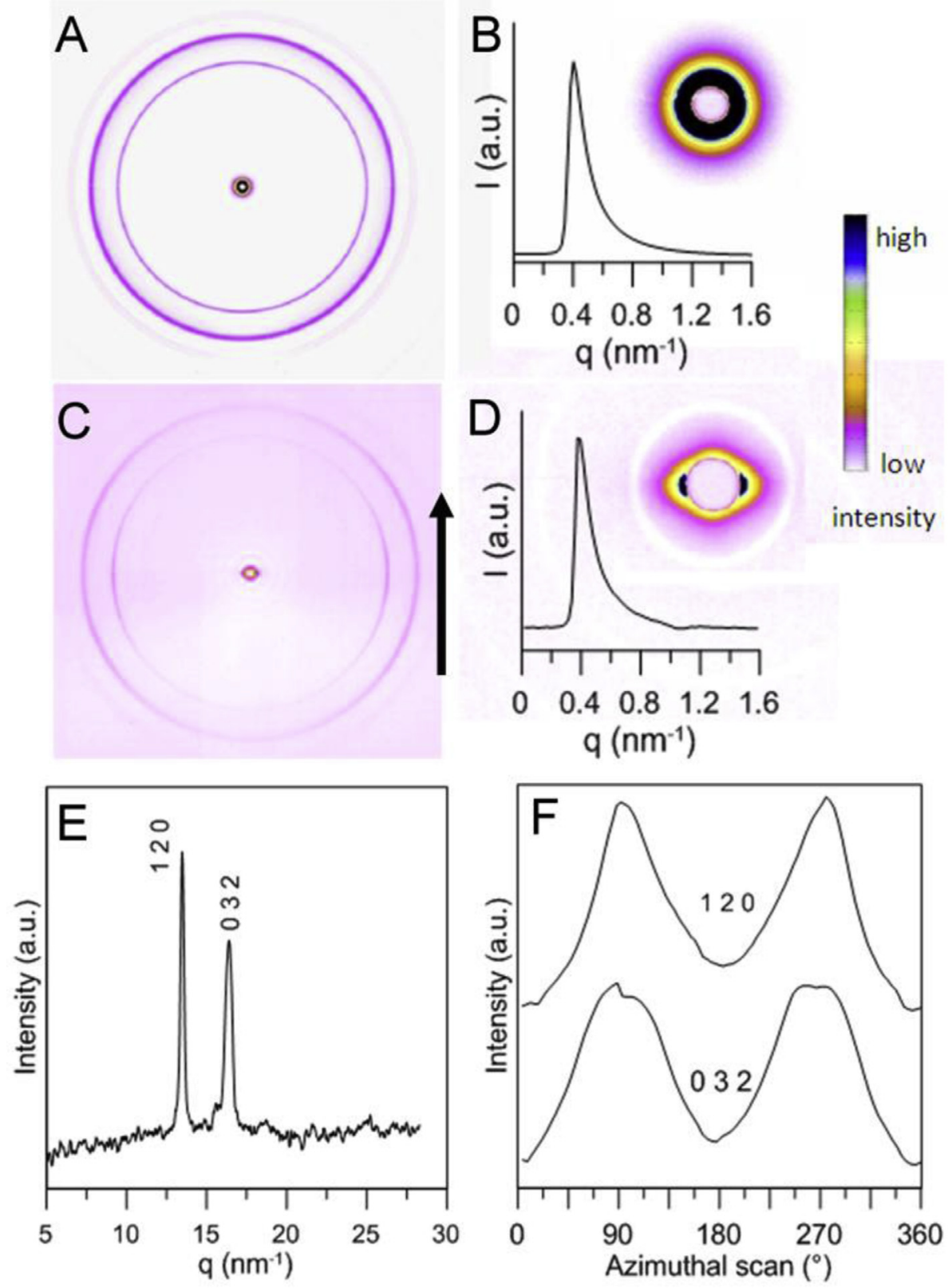

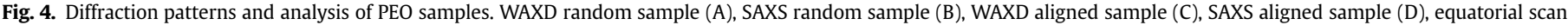

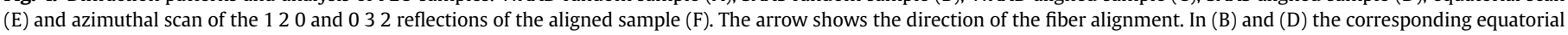
scans are reported.

intense wide angle reflections around the equator is higher than for the random samples (compare Fig. 4A and $\mathrm{C}$, Fig. 5A and $\mathrm{C}$ and Fig. $6 \mathrm{~A}$ and $\mathrm{C}$ ). The corresponding radial scans in the equator direction are reported in Figs. 4-6 panel E. Moreover, in the aligned samples the intensity distribution of the small angle diffusion shows an elliptical shape with the main axis on the equator direction while isotropic halo is seen in the random samples (compare Fig. 4B and D, Fig. 5B and D and Fig. 6A and D). These observations reveal that the crystal domains developed in the aligned fibers during electrospinning display mesoscopic preferred orientation.

The PEO pattern is well characterized by the 120 reflection at $d=0.465 \mathrm{~nm}$, as well as by the $032,-132,-212$ cluster of reflections at $d=0.383 \mathrm{~nm}$ that belong to the monoclinic cell structure that has been reported [38]. NYL pattern shows two partially overlapped 100 and 010 reflections of the $\alpha$-form [14,39], respectively at $d=0.432$ and $d=0.389 \mathrm{~nm}$. The two reflections of PAN at $d=0.522$ and $d=0.328 \mathrm{~nm}$ are indexed as 010 and 21 0 plus 300 , on the basis of the pseudo-hexagonal packing of solvent-free PAN molecules [40,41].

The crystal size (C.S.), i.e. the coherent scattering volume dimension in the direction perpendicular to the diffracting planes, is reported in Table 1, based on the estimates obtained using the Scherrer equation [42]. The fiber alignment does not affect this parameter, i.e. the results are similar for the random and aligned samples of the same material. PEO fibers show the biggest crystal size, almost three times that of NYL and PAN fibers, that themselves show similar values. The azimuthal scan of some intense peaks of the aligned samples is shown in Figs. 3-6F. From the width of the reflection it is possible to calculate the degree of parallelism ( $\Pi$ ) 

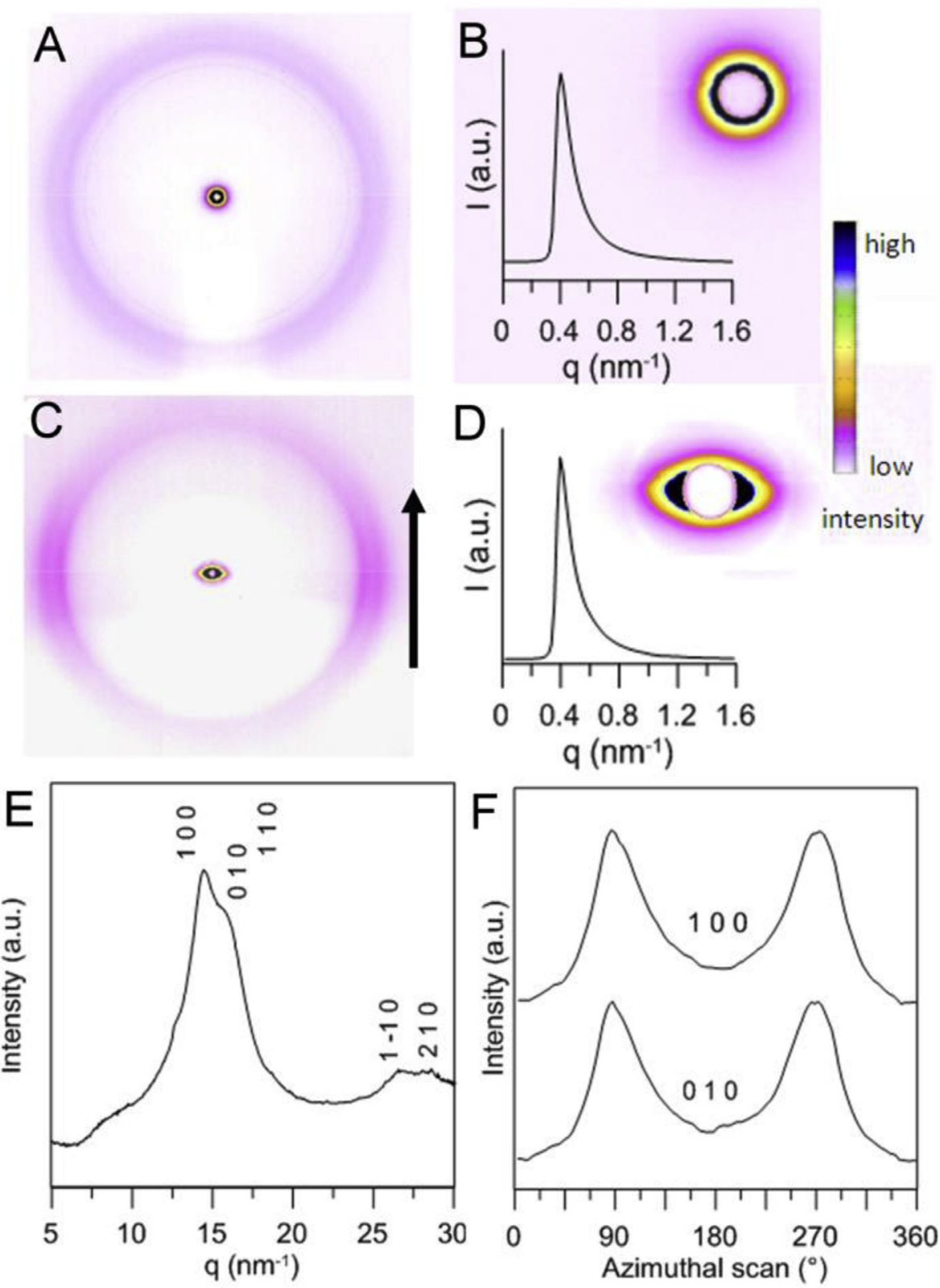

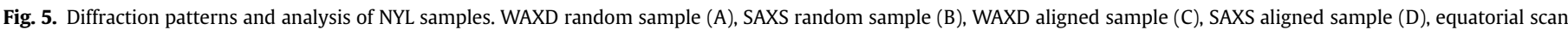

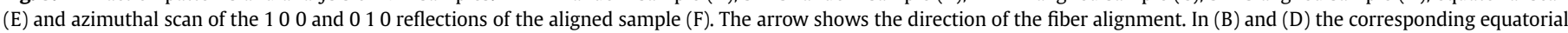
scans are reported.

[43], i.e. a parameter indicating the perfection of crystal orientation. All the aligned samples show a remarkably high value of $\Pi(58-73$, Table 1), indicating that the collection of electrospun fibers on a high-speed rotating drum resulted not only in the fiber alignment, but also led to a strong orientation of the crystalline domains and consequently to the molecular orientation in the crystalline portions of the fibers. It is pointed out that the observed spreading effect is the convolution of the scattering from the crystal domains in the fibers with the alignment distribution of the fibers themselves. As a consequence, the measurement of parallelism degree includes the effect of fiber angular distribution, but, since the alignment is high in all samples, the adjustment is proportionally negligible. The NYL sample possesses higher molecular orientation $(\Pi=73)$, again suggesting a possible role of hydrogen bonding in chain ordering during fiber processing. Indeed, it has been reported that NYL can assume a more perfect planar zig-zag conformation in the extended chain crystal structures under the influence of applied tensile stress [44]. This observation is confirmed by the increase of fusion enthalpy with respect to the pristine polymer previously discussed (Fig. 2C).

As far as SAXS analysis is concerned, random samples show isotropic strong diffuse scattering near the beam stop. Since the electrospun fibers are randomly oriented in the membrane, the pattern represents scattering from fibers in all directions. The treatment of small angle data for the random samples confirms that intensity $\mathrm{I}(\mathrm{q})$ scales with $\mathrm{q}^{-4}$ and the data can be treated under the Porod regime. Accordingly, the evaluation of the surface/volume ratio, without using any model for the shape of the crystals, indicates the presence of 'needle shaped' crystals, or unidirectionally extended fiber-like crystals. 

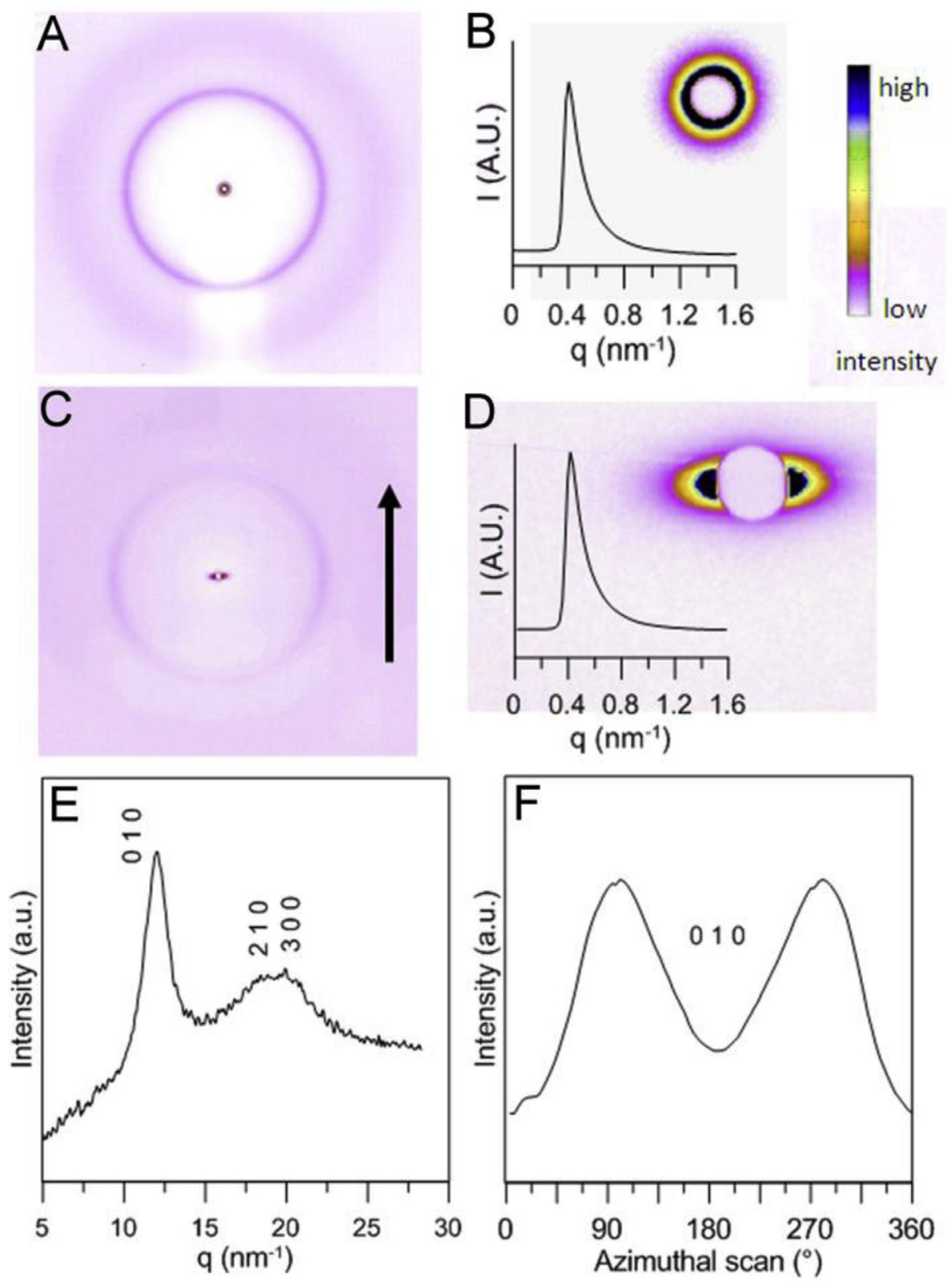

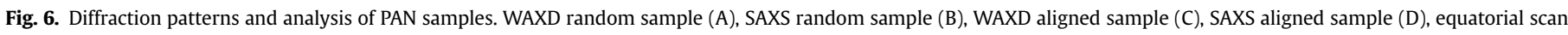

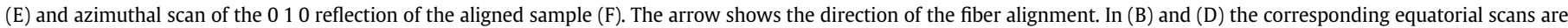
reported.

The SAXS patterns of PEO, NYL and PAN aligned samples have an elliptical shape with the main axis along the equator direction. No reflections are noticeable along the meridional direction suggesting that the investigated electrospun fibers have a fibrillar structure with no trace of lamellar morphology. Previous results demonstrated that fibers with smaller diameters have higher degree of crystallinity and molecular orientation in the form of fibrillar structure. This fibrillar structure is present together with densely packed aligned lamellae while with the increase of fiber diameter the electrospun fibers exhibit misaligned lamellae, without any fibrillar structure $[16,19]$. It may be hypothesized that the formation of oriented nuclei during the electrospinning process induces crystallization of polymer chains combined with extra elongation of the jet. As a result, smaller fibers are formed, with fibrillar crystals promoted by the stretching and drawing effect, that improves the degree of molecular orientation.
The interpretation of the equatorial spreading in the SAXS patterns of aligned fibers is more complicated since, in addition to the intensity due to the microfibrillar structure of the crystal, the needle-shaped morphology of the microvoids and the surface reflection of the fibers could give a further contribution to the spreading effect. The SEM observation of the folded and pressed samples prepared for the analysis, as reported in the experimental section, confirms the absence of microvoids.

In order to obtain information about the molecular organization that leads to asymmetrical scattering in the small angle region we applied the well tested method proposed by Ruland $[45,46]$. According to this method, the intensity at the equator is produced by scattering objects extended along the fiber direction. If the objects are strictly parallel to the fiber direction and have the same length $\mathrm{L}$, then the angular width of the diffusion halo is constant and not a function of the scattering angle. However, in most cases, as for the 


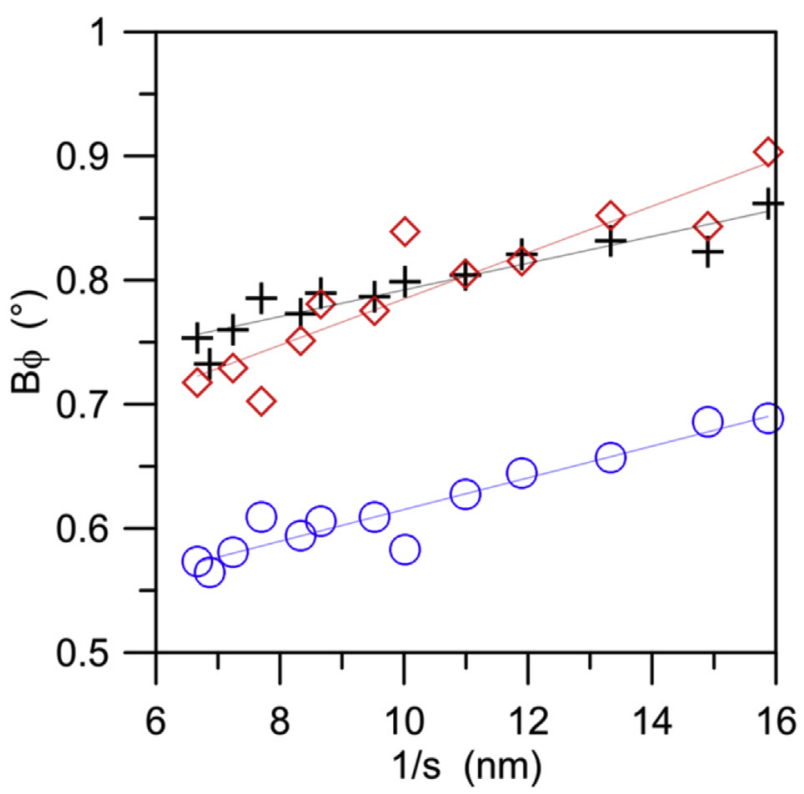

Fig. 7. Plot of $B_{\Phi}$ vs $1 / \mathrm{s}$. PEO (square), NYL (cross) and PAN (circle). The lines are the linear least square fits. $S=q / 2 \pi$.

samples here investigated, both the size distribution and the misorientation of the domains contribute to the intensity profile. A series of azimuthal scans at different $q$ values along the equator were performed. The integral breadth was determined as a function of the $q$ value.
As the relation $B_{o b s}=1 / L_{f} \cdot S+B_{\Phi}$ exists between the fibril length $\left(L_{f}\right)$ and the misorientation width $\left(B_{\Phi}\right)$, from the plot of $B_{\Phi}$ vs $1 / s$ (Fig. 7) it is possible to calculate these parameters (reported in Table 1). The length of the domain $\left(L_{f}\right)$ along the fiber axis is 93 and $78 \mathrm{~nm}$ for NYL and PAN samples, respectively and it is significantly shorter for PEO $\left(L_{f}=54 \mathrm{~nm}\right)$. The misorientation values $\left(B_{\Phi}\right)$, display low values for all the samples with no significant differences between them.

Combining the diffraction data with SEM observations it is possible to derive a sketch of the hierarchical structure of the electrospun fibers from the macro-to the nano-scale, as reported in Fig. 8. The NYL fibers show the smallest diameter and the highest molecular orientation among the investigated systems. NYL fibrillar crystals are thin and combining the crystal size (C.S.) data, estimated by the Scherrer equation, with structural information [39] we estimate that the ordered domains are organized in bundles of fibrils due to the lateral aggregation of roughly 10 molecular chains (10 unit cells). In the PEO sample the fiber diameter is about $50 \%$ bigger than NYL, but the degree of parallelism is lower. The crystallinity and the C.S. show the highest values indicating that in PEO fibers, about 20 molecular chains (10 unit cells) are aggregated in a fibril. PAN fibers have the biggest diameter, but they are formed by thin fibrils with lateral dimension similar to that observed in the NYL ones, deriving from the lateral aggregation of about 8 molecular chains (around 8 unit cells). In this case, the big fiber diameter and the fibril misalignment result in poor parallelism. The extent of crystal domains in the chains direction as estimated from the $\mathrm{L}_{\mathrm{f}}$ parameter is higher for NYL and PAN samples (about 50 unit cells) with respect to PEO sample (25 cells). Overall the observed ratio radius/height of the fibrils is small, indicating elongated fibrillar structures as reported by other authors $[10,16]$.
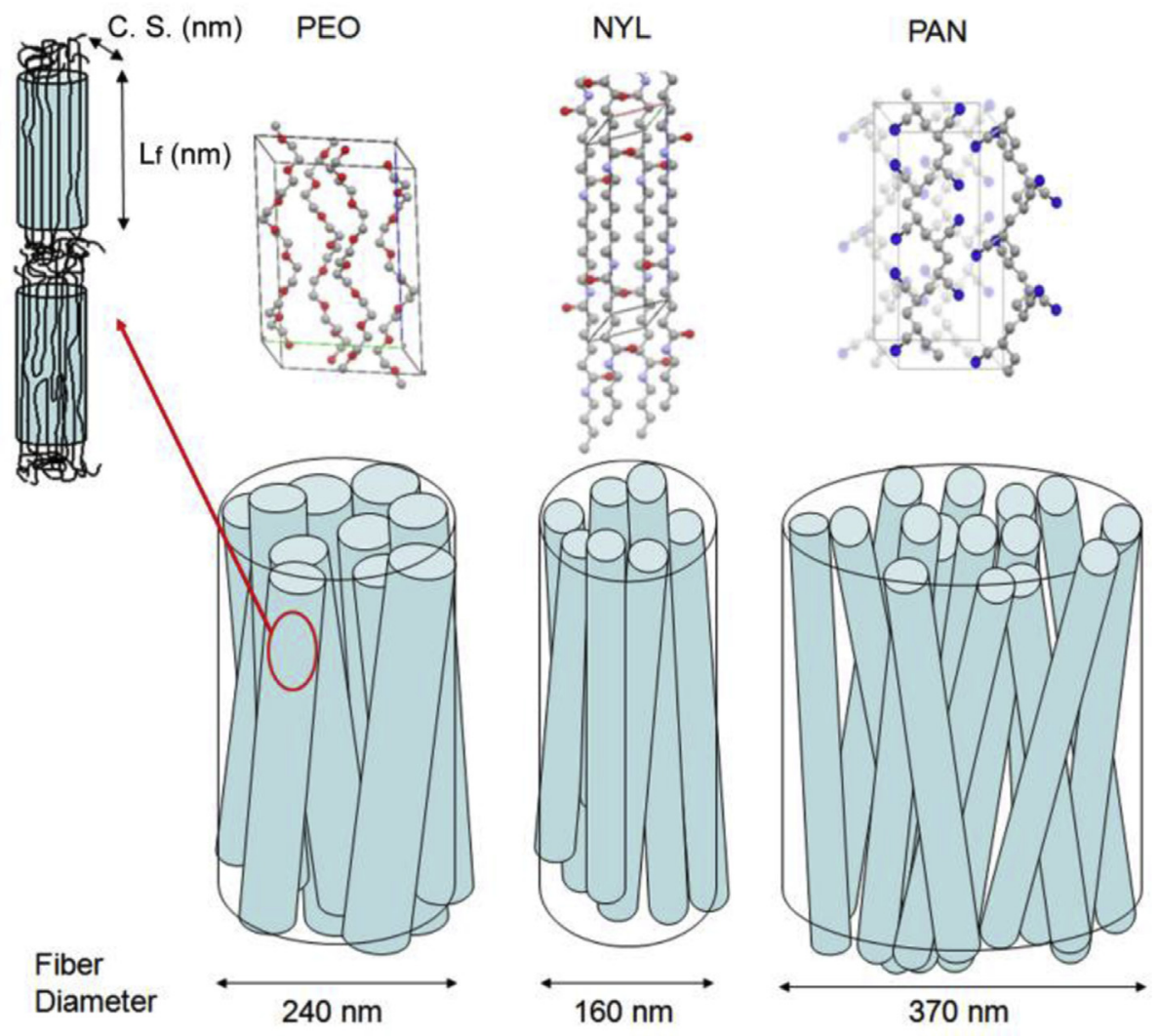

Fig. 8. Sketch of the structure-morphology relationship in electrospun fibers. 


\section{Conclusions}

Six samples of PEO, NYL and PAN nanofibers were prepared by solution electrospinning. The selection of favorable conditions allowed producing defect-free fibers with uniform diameters. The good crystallinity and the presence of the crystal phase were evidenced by DSC and X-ray analysis. The regular decrease of the diffuse intensity and the absence of any appreciable small angle reflection in X-ray diagrams suggests that under the electrospinning conditions used, fibrillar structures elongated in one direction were formed inside all the samples. A correlation between the morphology and the microstructure is proposed here that combines structural analysis with morphological and calorimetric data. The high degree of orientation of the crystals and consequently the high level of molecular alignment achieved for the NYL sample suggests a possible contribution of the electrospinning process for promoting the conformation that is most suitable for directional polymer crystallization.

\section{Acknowledgments}

The Authors acknowledge the Italian Ministry of University and Research for the financial support. MLF acknowledges the support of FP7 COST Action MP1206 "Electrospun Nano-fibres for bio inspired composite materials and innovative industrial applications". AMK acknowledges the provision of laboratory space and facilities for the Centre for In situ Processing Studies (CIPS) at the Research Complex at Harwell, and beamtime allocation EE6634 by the Diamond Light Source. AMK also acknowledges funding for the Oxford MBLEM laboratory received through the EU FP7 project iSTRESS (604646), and EPSRC support through grants EP/I020691/1, EP/G035059/1, EP/G004676/1 and EP/H003215/1.

\section{References}

[1] Greiner A, Wendorff JH. Angew Chem Int Ed 2007;46:5670-703.

[2] Ramakrishna S, Fujihara K, Teo W-E, Lim T, Ma Z. An introduction to electrospinning and nanofibers. Singapore: World Scientific Publishing; 2005.

[3] Baji A, Mai YW, Wong SC, Abtahi M, Chen P. Compos Sci Technol 2010;70: 703-18.

[4] Richard-Lacroix M, Pellerin C. Macromolecules 2013;46:9473-93.

[5] Doshi J, Reneker DH. J Electrost 1995;35:151-60.

[6] Ward IM. Developments in oriented polymers. London: Applied Science Publishers; 1975.

[7] Ward IM. Developments in oriented polymers-2. London: Applied Science Publishers; 1987.

[8] Reynolds N. Adv Mater 1991;3:614-6.
[9] Kakade MV, Givens S, Gardner K, Lee KH, Chase DB, Rabolt JF. J Am Chem Soc 2007; $129: 2777-82$

[10] Kongkhlang T, Tashiro K, Kotaki M, Chirachanchai S. J Am Chem Soc 2008;130: 15460-6.

[11] Lee KH, Kim KW, Pesapane A, Kim HY, Rabolt JF. Macromolecules 2008;41: 1494-8.

[12] Dror Y, Salalha W, Khalfin RL, Cohen Y, Yarin AL, Zussman E. Langmuir 2003; 19:7012-20.

[13] Edwards MD, Mitchell GR, Mohan SD, Olley RH. Eur Polym J 2010;46: $1175-83$.

[14] Zussman E, Burman M, Yarin AL, Khalfin R, Cohen Y. J Polym Sci Part B Polym Phys 2006;44:1482-9.

[15] Yano T, Higaki Y, Tao D, Murakami D, Kobayashi M, Ohta N, et al. Polymer 2012;53:4702-8.

[16] Lim CT, Tan EPS, Ng SY. Appl Phys Lett 2008;92:141908.

[17] Jaeger R, Schönherr H, Vancso GJ. Macromolecules 1996;29:7634-6.

[18] Huang C, Chen S, Lai C, Reneker DH, Qiu H, Ye Y, et al. Nanotechnology 2006; 17:1558-63.

[19] Yoshioka T, Dersch R, Tsuji M, Schaper AK. Polymer 2010;51:2383-9.

[20] Ma X, Liu J, Ni C, Martin DC, Chase DB, Rabolt JF. ACS Macro Lett 2012;1: 428-31.

[21] Bellan LM, Craighead HG. Polymer 2008;49:3125-9.

[22] Pagliara S, Vitiello MS, Camposeo A, Polini A, Cingolani R, Scamarcio G, et al. J Phys Chem C 2011;115:20399-405.

[23] Hobson RJ, Windle AH. Macromolecules 1993;26:6903-7.

[24] Ayres CE, Jha BS, Meredith H, Bowman JR, Bowlin GL, Henderson SC, et al. J Biomater Sci Polym Ed 2008;19:603-21.

[25] Wunderlich B. Thermal analysis of polymeric materials. The Netherlands: Springer-Verlag Berlin Heidelberg; 2005.

[26] http://www.esrf.eu/computing/scientific/FIT2D/.

[27] Förster S, Apostol L, Bras W. J Appl Crystallogr 2010;43:639-46.

[28] Focarete ML, Gualandi C, Scandola M, Govoni M, Giordano E, Foroni L, et al. J Biomater Sci Polym Ed 2010;21:1283-96.

[29] Gualandi C, Govoni M, Foroni L, Valente S, Bianchi M, Giordano E, et al. Eur Polym J 2012;48:2008-18.

[30] Kim KW, Lee KH, Khil MS, Ho YS, Kim HY. Fibers Polym 2004;5:122-7.

[31] Zhong X, Kim K, Fang D, Ran S, Hsiao BS, Chu B. Polymer 2002;43:4403-12.

[32] Deitzel JM, Kleinmeyer JD, Hirvonen JK, Beck Tan NC. Polymer 2001;42: 8163-70.

[33] Zhong X, Ran S, Fang D, Hsiao BS, Chu B. Polymer 2003;44:4959-67.

[34] Grassie N, McGuchan R. Eur Polym J 1970;6:1277-91.

[35] Hou X, Yang X, Zhang L, Waclawik E, Wu S. Mater Des 2010;31:1726-30.

[36] Lai C, Zhong G, Yue Z, Chen G, Zhang L, Vakili A, et al. Polymer 2011;52: 519-28.

[37] Gu SY, Ren J, Wu QL. Synth Met 2005;155:157-61.

[38] Takahashi Y, Tadokoro H. Macromolecules 1973;6:672-5.

[39] Bunn CW, Garner EV. Proc R Soc Lond Ser A Math Phys Sci 1947;189:39-68.

[40] Bashir Z. Polymer 1992;33:4304-13.

[41] Bashir Z. Acta Polym 1996;47:125-9.

[42] Alexander LE. X-ray diffraction methods in polymer science. New York: Wiley-Interscience; 1969.

[43] Kakudo N, Kasai N. X-ray diffraction by polymers. Amsterdam: Elsevier; 1972.

[44] Cho D, Zhmayev E, Joo YL. Polymer 2011;52:4600-9.

[45] Ran S, Fang D, Zong X, Hsiao BS, Chu B, Cunniff PM. Polymer 2001;42: $1601-12$.

[46] Ruland W. J Polym Sci Part C Polym Symp 1969;28:143-51. 